\title{
Sphingosine 1-phosphate and Cancer
}

Nigel J. Pyne*, Ashref El Buri, David R. Adams ", Susan Pyne

Strathclyde Institute of Pharmacy and Biomedical Sciences, University of Strathclyde, 161

Cathedral St, Glasgow, G4 0RE, Scotland, UK. // Institute of Chemical Sciences, HeriotWatt University, Edinburgh, EH14 4AS, Scotland, UK

* To whom correspondence should be addressed (email: n.j.pyne@strath.ac.uk)

Key words: Sphingosine 1-phosphate, sphingosine kinase, sphingosine 1-phosphate receptors, cancer cell-fibroblast interactions, metastasis.

Abstract--The bioactive lipid, sphingosine 1-phosphate (S1P) is produced by phosphorylation of sphingosine and this is catalysed by two sphingosine kinase isoforms (SK1 and SK2). Here we discuss structural functional aspects of SK1 (which is a dimeric quaternary enzyme) that relate to coordinated coupling of membrane association with phosphorylation of Ser225 in the 'so-called' R-loop, catalytic activity and protein-protein interactions (e.g. TRAF2, PP2A and $\mathrm{G}_{\mathrm{q}}$ ). S1P formed by SK1 at the plasma-membrane is released from cells via S1P transporters to act on S1P receptors to promote tumorigenesis. We discuss here an additional novel mechanism that can operate between cancer cells and fibroblasts and which involves the release of the $\mathrm{S} 1 \mathrm{P}$ receptor, $\mathrm{S}_{1} \mathrm{P}_{2}$ in exosomes from breast cancer cells that regulates ERK-1/2 signalling in fibroblasts. This novel mechanism of signalling might provide an explanation for the role of $\mathrm{S} \mathrm{P}_{2}$ in promoting metastasis of cancer cells and which is dependent on the micro-environmental niche. 
Introduction--The bioactive lipid, sphingosine 1-phosphate $(\mathrm{S} 1 \mathrm{P})$ is produced by phosphorylation of sphingosine using ATP and this is catalysed by two sphingosine kinase isoforms (SK1 and SK2). These enzymes are encoded by distinct genes and differ in their subcellular localisation, biochemical properties and functions. S1P can either be exported from cells to bind to a family of five S1P-specific $\mathrm{G}$ protein coupled receptors $\left(\mathrm{S}_{1} \mathrm{P}_{1-5}\right)$ or can interact with specific intracellular target proteins, such as human telomerase reverse transcriptase (hTERT), to induce cell responses (Pyne et al., 2016). A role for S1P in cancer is suggested from studies demonstrating that high expression of SK1, SK2 and S1P receptors in tumours is linked with poor prognosis in patients (Watson et al., 2010; Ohotski et al., 2013; Wang et al., 2014a). There is also substantial evidence for a role for S1P in promoting transformation, epithelial mesenchymal transition and invasiveness, cancer cell survival, replicative immortality, tumour neovascularisation and aerobic glycolysis; the so-called hallmarks of cancer (Pyne and Pyne, 2010; Pyne et al., 2016; Maczis et al., 2016). Therefore, both SK1 and SK2 are potential targets for therapeutic intervention in cancer. Transformation of NIH3T3 cells into fibrosarcoma can be achieved by over-expression of SK1 (Xia et al., 2000) and this involves its translocation from the cytoplasm to the plasma-membrane, a process that allows access to substrate (sphingosine). In this regard, cancer cells exhibit a nononcogenic addiction for SK1 (Vadas et al., 2008). Indeed, over-expression of a kinase-dead G82D SK1 mutant fails to induce transformation, thereby demonstrating dependency on the 
catalytic activity of SK1 and therefore on S1P (Xia et al., 2000). A key step in the transformation process is the phosphorylation of SK1 by extracellular regulated kinase (ERK) on Ser225, which promotes translocation of the enzyme from the cytoplasm to the plasmamembrane (Pitson et al., 2003; Pitson et al., 2005). Indeed, constitutive localisation of a phosphorylation deficient mutant of SK1 to the plasma-membrane promotes so called oncogenic signalling (Pitson et al., 2005). Moreover, recent studies have demonstrated that SK1 activation and localization to the plasma membrane and subsequent activation of $\mathrm{S}_{1} \mathrm{P}_{2}$ by released S1P ('inside-out' signalling) increases transferrin receptor 1 (TFR1) expression (Pham et al., 2014). Moreover, inhibition of TFR1 with a neutralising antibody prevents SK1-induced proliferation, survival and neoplastic transformation of NIH3T3 fibroblasts (Pham et al., 2014). In addition, recent evidence demonstrates a role for SK1 in endosomal processing (Shen et al., 2014). In this regard, depletion of cholesterol/sphingomyelin in the plasma membrane induces the formation of clusters of narrow endocytic tubular invaginations that are positive for N-BAR proteins and SK1 in various mammalian cell lines (Shen et al., 2014). The association with early endosomes occurs via a hydrophobic patch located in the lipid binding loop-1 (LBL-1) of SK1 and this occurs in a curvature-sensitive manner (vide infra). Moreover, knockdown of SK1 produces endocytic recycling defects (Shen et al., 2014). The regulation of endocytic recycling by SK1 might, therefore, impact significantly on disease forming mechanisms in cancer. Taken together, these findings suggest that the redistribution of SK1 from the cytoplasm to the plasma-membrane/endosomes requires more thorough understanding from a structural/functional perspective.

In this review, we discuss structure-function aspects of SK1 that relate to its regulation and plasma-membrane association. SK1 adopts a dimeric quaternary structure and this organisation, as we detail below, may allow for coordinated coupling of membrane association 
with phosphorylation of Ser225 in the so-called 'R-loop', catalytic activity and protein-protein interactions (e.g. TRAF2, PP2A and $\mathrm{G}_{\mathrm{q}}$ ). Plasticity in the ATP binding domain appears to be important for catalytic operation and is likely modulated by a helix-capping mechanism involving the regulatory C-terminal protein-binding sequence, also discussed below (Adams et al., 2016).

Current established models for the action of S1P involve its release and binding to S1P receptors on recipient cells (Takabe and Spiegel, 2014). This underlies inter-cellular communication between, for instance, cancer cells and fibroblasts in the micro-environmental niche that can promote metastatic spread. Multiple S1P receptors can be expressed in a single cell type, and it is currently unclear how S1P signalling through these receptors is differentiated to induce specific S1P receptor-mediated cellular response programmes. One possibility is that S1P partitions to the plasma-membrane and is carried by chaperones in a controlled process that allows its delivery to specific receptors (Galvani et al., 2015). However, we discuss here an additional novel mechanism that can operate between cancer cells and fibroblasts and which involves release of the $\mathrm{S} 1 \mathrm{P}$ receptor, $\mathrm{S}_{1} \mathrm{P}_{2}$, in exosomes from breast cancer cells (unpublished). The released $\mathrm{S} 1 \mathrm{P}_{2}$ is then proteolysed by fibroblasts to produce a constitutively active form (S1P independent) that regulates the ERK-1/2 pathway in fibroblasts and promotes their proliferation. This novel mechanism of signalling might provide an explanation for the role of $\mathrm{S} \mathrm{P}_{2}$ in promoting metastasis of cancer cells and which involves expansion of the fibroblast population.

Structural features of sphingosine kinase 1--SK1 has a two-domain structure with an Nterminal domain (NTD) hosting the binding site for ATP and a C-terminal domain (CTD) containing the binding site for sphingosine (Figure 1) (Wang et al., 2013). The inter-domain 
junctional cleft hosts the catalytic centre. The NTD is composed of an $\alpha / \beta / \alpha$ sandwich containing six $\beta$-strands $(\beta 1-\beta 5$ and $\beta 17)$ and six $\alpha$-helices $(\alpha 1-\alpha 6)$. The CTD domain is organised into a two-layer $\beta$-sandwich containing $11 \beta$-strands $(\beta 6-\beta 16)$. Three extended loops between strands $\beta 8-\beta 9, \beta 10-\beta 11$ and $\beta 11-\beta 12$ contribute to the sphingosine (Sph) binding site. These are called lipid binding loops 1-3 (LBL-1 to LBL-3). LBL-1 folds with two reverse-paired helices $(\alpha 7-\alpha 8)$, with the inter-helix connection positioned into the catalytic cleft. The shorter LBL-2 interfaces with the NTD at the catalytic cleft and lacks secondary structure. LBL-3 contains an additional helix ( $\alpha 9)$ that runs antiparallel along $\alpha 8$ and does not make direct contact with the NTD. The binding site for sphingosine lies between $\alpha 7-\alpha 9$ and interfaces with the $\beta$-sandwich of CTD. The reverse face of this $\beta$-sandwich contains a regulatory loop ('R-loop') which is present between strands $\beta 9 / \beta 10$ and contains the ERK phosphorylation site at Ser225 (Pitson et al., 2003).

The nucleotide binding site in SK1 involves a number of key structural motifs (Figure 1, inset) (Wang et al., 2013). The adenine makes contact with residues 22-NPR-24 (motif-1) and 54-TERR-57 (motif-2). A third motif (79-SGDGLMHE-86, motif-3) makes contact with the $\alpha$-phosphate of the nucleotide and is often referred to as the 'P-loop'. The $\beta$-phosphate is engaged by motif-4 (111-GSGNAL-116), referred to as the 'T-loop'. A catalytic $\mathrm{Mg}^{2+}$ ion, that coordinates to the nucleotide phosphates, is bound to a DGE sequence (motif-5) through the carboxylate of Glu343 and backbone carbonyl of Asp341. In SK1 crystal structures with bound ADP, the $\beta$-phosphate is seen to interact with Arg185 and Arg191 present in LBL-1. However, Arg191 might potentially be involved in binding the ATP $\gamma$-phosphate and the S1P product during actual catalytic operation. 
Sphingosine is accommodated in the so-called ' $\mathrm{J}$-channel', where there is surface contact with hydrophobic residues in LBL-1-3 and across the face of the CTD $\beta$-sandwich core. The Sph-1-OH is positioned in loose contact with P-loop Asp81 (Figure 1, inset), which serves as the base to deprotonate sphingosine and allow transfer of the $\gamma$-phosphate from ATP by nucleophilic attack (Wang et al., 2013). The Sph-3-OH engages Asp178 on LBL-1 to anchor the substrate head group in the mouth of the J-channel.

Regulation of plasma-membrane association of SK1--Transformation of normal cells into cancer cells by SK1 involves the enzyme's translocation from the cytoplasm to the plasmamembrane, where it can access its substrate, sphingosine. There are multiple factors that govern the translocation of SK1 to the plasma-membrane and its subsequent residence there. One regulatory mechanism involves the phosphorylation of Ser225 in SK1 by ERK, which promotes both membrane targeting and enhanced activity of the enzyme (Pitson et al., 2003; Pitson et al., 2005)]. Interactions with acidic phospholipids, notably with phosphatidylserine (PS) (Olivera et al., 1996; Stahelin et al., 2005), also play a key role in the activation and membrane localisation of SK1. It has been proposed that the enzyme hosts a specific recognition site for the lipid head group of PS that might potentially be exposed upon phosphorylation of Ser225 by ERK. In particular, Thr54 and Asn89 have been identified as residues in SK1 that are important for interaction of the enzyme with PS at the plasmamembrane (Stahelin et al., 2005; Hengst et al., 2009), although it is not known whether the residues themselves interact directly with PS.

The structural change in SK1 elicited by phosphorylation of Ser225 in the R-loop has yet to be defined. Curiously, in the available SK1 crystal structures (Wang et al., 2013; Gustin et al., 2013; Wang et al., 2014b), Ser225 is seen to occupy a prominently solvent-exposed 
position (Figure 2). A regulatory mechanism in which phosphorylation simply lifts the Rloop from the surface of the protein appears inconsistent with this pre-exposed position for Ser225. Clues as to the possible structural impact of the phosphorylation are, however, suggested by the observed interaction of the R-loop with the NTD of SK1. Thus, in the available crystal structures, when the tip of the R-loop is ordered it is found packed against a sequence (93-ERPDW-97) located at the $\alpha-3 / 4$ junction in the NTD (Figure 2). R-loop movement induced by phosphorylation may therefore conceivably perturb this CTD-NTD interface. Asn89, one of the residues known to be important for targeting of SK1 to PSenriched sites (Stahelin et al., 2005), is proximally located on helix- $\alpha 3$ and also makes a key cross-domain contact in the form a doubly hydrogen bonded interaction across the interdomain cleft to the backbone of Phe164 on CTD $\beta 7$. Taken together, these considerations suggest that inter-domain movement and/or perturbation of residues distributed across $\alpha 3 / 4$ may be important to the interaction with PS and therefore to membrane residence of SK1. One possible mechanism by which phosphorylation might elicit such a perturbation could involve displacement of R-loop aspartate, Asp235, from a basic pocket on the SK1 CTD lined by His156/Arg162/His355 (Adams et al., 2016). Crystal structures suggest that docking of Asp235 into this site (Figure 2) likely plays an important role in stabilising the basal state R-loop conformation and cross-domain interaction with the $\alpha-3 / 4$ junction. The position of Ser225 is such that in its phosphorylated state it might potentially target the Asp235 binding locus, which would then displace the R-loop tip from its NTD engagement. We have proposed (Adams et al., 2016) that conformational relaxation in the SK1-ERPD sequence at the $\alpha-3 / 4$ junction modulates a region of positive electrostatic potential surface proximal to the SK1 dimerisation interface ( $c f$. Figure 3) and to Thr54 (a residue also known to be important for the interaction of SK1 with PS). 
Whilst residence of the enzyme at the plasma-membrane may be subject, at least in part, to interaction with acidic phospholipids, the translocation process itself may be facilitated by additional factors. In particular, association with calcium and integrin-binding protein 1 (CIB1) has been shown to play a role in the enzyme's translocation (Jarman et al., 2010). Intriguingly, surface-exposed hydrophobic residues (Phe197/Leu198) on helix- $\alpha 8$ of LBL-1, that have been implicated in the interaction of SK1 with CIB1, may also be involved in the direct interaction of SK1 with the membrane itself following translocation, potentially by immersion within the membrane. Thus, mutation of these residues or (separately) the adjacent Leu194, so as to compromise an exposed hydrophobic patch on helix-a8 (Figure 3), blocks binding of SK1 both to acidic liposomes in vitro and curvature-sensitive membrane association of SK1 in living cells (Sutherland et al., 2006; Shen et al., 2014). This suggests that membrane residence may involve a combination of polar interactions between SK1 and acidic membrane phospholipids augmented by insertion of LBL-1 into the membrane.

Such a model raises questions about the overall topology of the protein and its approach to the membrane surface. We have reported that SK1 adopts a functionally significant dimeric quaternary structure (Lim et al., 2011), which likely corresponds to the dimeric assembly seen in all five of the currently available SK1 crystal structures. Dimerisation in the crystal lattices involves partial annealing of the exposed $\beta 2$ strands (Figure 1) from two adjacent protein subunits to generate a C2-symmetric NTD-NTD interface that also involves interactions with residues in the helices that flank $\beta 2$ on either face. A common pattern of head-to-head dimerization is widely seen across structurally related prokaryotic lipid kinase enzymes belonging to the diacylglycerol kinase DAGK_cat family (Pfam 00781) (Adams et al., 2016). In the case of SK1, this mode of dimerisation generates a pronounced groove at the dimer interface which is aligned parallel with the helix- $\alpha 8$ axes from the two LBL-1 loops 
of the dimer, these being offset on either side of the central groove (Figure 3). The groove exhibits significant net positive charge density and a structure that might potentially be sensitive to modulation by phosphorylation of the R-loop according to the 'relay mechanism' postulated in Figure 2. Significantly, the exposed helix- $\alpha 8$ hydrophobic surfaces map to the same face of the SK1 dimer so that docking of the positively charged groove to the membrane surface might allow simultaneous insertion of the LBL-1 loops into the membrane. An intriguing consequence of the dimeric structure with the central groove, therefore, is that topologically the protein might be optimally suited for binding in a dimeric state to a membrane surface exhibiting positive curvature, as illustrated in Figure 3 panel A (ii). Such a model could then account for the findings of Shen et al., (2014), who have recently reported that membrane recruitment of SK1 in living cells involves a direct, curvature-sensitive interaction with the lipid bilayer and a pronounced preference for regions of high positive curvature, notably at narrow tubular membrane invaginations thought to correspond to early endocytic structures. We have therefore proposed that SK1 recruitment to such structures may be facilitated by alignment of the dimer cleft along the curvature axis (Adams et al., 2016). An important corollary of the proposed membrane engagement mode (Figure 3) is that it also provides a plausible rationale for Sph substrate extraction from the membrane by a gating mechanism in which helices-7/8 of LBL-1 open to expose the hydrophobic surface of the J-channel and then reclose to encapsulate the substrate, thus isolating the Sph head group within the enzyme's interior for selective phosphoryl transfer from ATP.

C-terminal regulation of SK1 activity and translocation to the plasma-membrane--The Cterminal tail of SK1, which is known to support the binding of partner proteins (vide infra), is likely to play an important role in regulating SK1 with potential ramifications for cancer disease progression. Indeed, truncation after residue 363 in the C-terminal tail renders SK1 
constitutively active and promotes localization to PS-containing lipid raft micro-domains (without requirement for phosphorylation of R-loop Ser225) (Hengst et al., 2010). Remarkably, the addition of just four residues (364-367) is sufficient to restore the lower basal activity of full-length SK1 but in a manner that does not depend on residue identity, suggesting a role for the peptide backbone of this region in modulating catalytic activity. All of the currently available SK1 crystal structures are truncated, terminating at Gly364, so that the structure and folding of the enzyme's C-terminal 20 residues is currently unknown. Nevertheless, inspection of the crystal structures does provide clues as to the possible mechanism of action of this important sequence region in regulating SK1.

Gly364 is located immediately adjacent to the exposed C-terminus of the short helix- $\alpha 5$, at the opposite end to the so-called T-loop (Figure 3A), which is involved in ATP binding and catalytic activity. Residues $364-367$ are therefore predicted to be suitably positioned for capping of helix- $\alpha 5$ by means of backbone hydrogen donor interactions (Figure 1 inset, black arrows). Any leverage exerted on helix- $\alpha 5$ by modulation of this proposed helix capping arrangement might then be relayed into the catalytic centre to regulate activity. Comparison of SK1 crystal structures with and without bound nucleotide reveals significant changes in the conformation of T-loop residues. Thus, plasticity in the T-loop may be important for enzyme function, potentially allowing the T-loop to track the nucleotide $\beta$-phosphate with backbone hydrogen donor interactions on separation from the $\gamma$-phosphate of ATP during catalytic turnover (Adams et al., 2016). Leverage on the T-loop associated with modulated C-terminal capping of helix- $\alpha 5$ therefore provides a highly plausible mechanism for regulating catalytic activity, with $\mathrm{C}$-terminal capping of helix- $\alpha 5$ providing a 'braking' mechanism. Removal of the postulated helix cap could then satisfactorily account for the observed constitutive activation associated with C-terminal truncation at residue 363. 
The mechanism by which C-terminal truncation promotes localization to PS-enriched membrane structures has yet to be elucidated. One possibility is that, in the basal state, the Cterminal sequence folds in an auto-inhibitory manner to hinder presentation of the positively charged putative membrane engagement surface of SK1, for example by targeting of the Cterminal 379-PPEE-382 sequence to critical basic residues required for PS engagement. In this way movement of the C-terminal tail might orchestrate membrane association coordinated precisely with increased catalytic activity exerted by changes in capping of helix$\alpha 5$, and it follows that sequestration of the $\mathrm{C}$-terminal tail by binding proteins could thereby regulate both SK1 translocation to the plasma-membrane and catalytic activity. Alternatively, the C-terminus might obstruct dimerization, if the PS engagement is dependent on the dimeric assembly. The full extent to which proteins from the SK1 interactome exploit the C-terminal tail remains to be determined, but established examples include TRAF2 (tumour necrosis factor receptor-associated factor 2) (Xia et al., 2002) and PP2A (Barr et al., 2008), the protein phosphatase responsible for reversing phosphorylation of Ser225. These proteins have been shown, respectively, to bind to the 79-PPEE-382 sequence and to residues in the region 368-384. Another example of a possible SK1 tail-binding protein is $\mathrm{G}_{\mathrm{q}}$. Both wild type and constitutively activated forms of $\mathrm{G}_{\mathrm{q}}\left(\right.$ e.g. $\left.\mathrm{G} \alpha_{\mathrm{q}}{ }^{\mathrm{R} 183 \mathrm{C}}, \mathrm{G} \alpha_{\mathrm{q}}{ }^{\mathrm{Q} 209 \mathrm{~L}}-\mathrm{EE}\right)$ have been shown (ter Braak et al., 2009) to promote translocation of SK1 to the plasma-membrane independently of Ser225 phosphorylation and therefore, we suggest, perhaps by a mechanism that involves sequestration of the SK1 C-terminal tail. Whilst the $\mathrm{G}_{\mathrm{q}}$-binding determinants on SK1 remain to be confirmed, the physiological significance of the $\mathrm{SK} 1-\mathrm{G}_{\mathrm{q}}$ interaction is evident from studies showing that SK1 is involved in promoting neurotransmission in Caenorhabditis elgans (Chan et al., 2012). Thus, it has recently been demonstrated that SK1 localisation in presynaptic neuron periactive zones, which contain endocytic proteins for 
synaptic vesicle recycling, is increased by muscarinic receptor stimulation of $G_{q} /$ RhoGEF in C. elgans (Chan et al., 2012). Worms expressing an SK1 V268Q mutation, which corresponds to a mutation of LBL-1 L194 in mammalian SK1, exhibit a neurotransmission defect that is rescued by the expression of WT SK1. This occurs because the mutant SK1 fails to translocate to the periactive zone membrane due to the compromised LBL-1 (Shen et al., 2014).

Membrane targeting clearly plays a key role in the functioning of SK1 in cancer. The existence of both phosphorylation-dependent and -independent mechanisms for driving localization of SK1 to membranes raises a question as to whether there is any mechanistic intersection between the differing modes of localisation at the level of protein structure. This might occur, for example, if the outcome of Ser225 phosphorylation in the SK1 R-loop is to destabilise auto-inhibitory C-terminal folding so as to directly expose the enzyme's PSbinding determinants or allow their formation through dimer assembly. In this way, a unified mechanism of action for membrane localization might operate, driven either by phosphorylation of Ser225 to release the SK1 tail or by sequestration of the tail through direct protein-protein interactions. If the interaction with PS is dependent on the dimeric assembly of SK1, then potentially the SK1 tail (in its basal state) might obstruct dimer assembly.

The proposed model for membrane engagement of dimeric SK1 (Figure 3) positions the two Gly364 tail connection sites on either side of the protein assembly. As such, the tail sequence is predicted to be able to run clear of the membrane surface and to be able to accommodate protein binding with the enzyme operationally engaged at the membrane surface. This is required for localization and activation driven by protein binding to the SK1 tail rather than by phosphorylation. 
$\mathrm{SIP}_{3}$ receptor-dependent translocation of $\mathrm{SK1--We}$ have demonstrated a key role of $\mathrm{S} 1 \mathrm{P}_{3} / \mathrm{SK} 1$ in regulating the formation of a migratory phenotype in MCF-7 cells (Long et al., 2010). S1P binding to $\mathrm{S}_{3} P_{3}$ stimulates activation of the ERK-1/2 pathway, and phosphorylated ERK-1/2 accumulates in membrane ruffles/lamellipodia and the nucleus to promote MCF-7 cell migration. We also demonstrated that S1P promotes the translocation of SK1 from the cytoplasm to the plasma-membrane and that this was blocked by an $\mathrm{S}_{1} \mathrm{P}_{3}$ antagonist (Long et al., 2010). Since $\mathrm{S}_{1} \mathrm{P}_{3}$ is coupled to $\mathrm{G}_{\mathrm{q}}$, it is possible that this G-protein is involved in regulating the translocation of SK1 to the plasma-membrane of breast cancer cells. These findings are intriguing as they suggest that $\mathrm{S} 1 \mathrm{P}$ binding to $\mathrm{S}_{3} \mathrm{P}_{3}$ on the cell surface is transduced into an intracellular S1P signal, which, we propose, regulates the phosphorylation and activation of PAK65, which is required for actin/phosphorylated ERK1/2 redistribution into lamellipodia and cell migration (Long et al., 2010). We have also reported that the siRNA knockdown of $\mathrm{SK} 1$ reduces $\mathrm{S}_{1} \mathrm{P}_{3}$ mRNA transcript expression in MCF-7 cells and that this ablates S1P-stimulated activation of ERK-1/2 in response to S1P (Long et al., 2010). siRNA knockdown of SK1 also ablated the S1P-stimulated formation of actin containing membrane ruffles/lamellipodia and restored the formation of focal actin adhesion contacts and inhibited migration. Therefore, SK1 appears to act as a sensor that regulates the amplitude of $\mathrm{S}_{1} \mathrm{P}_{3}$ receptor signalling. The $S 1 P R 3$ gene contains predicted ER, Sp1, and c-Jun binding sites in its promoter. Sp1 and c-Jun are regulated by ERK-1/2, and therefore this protein kinase might be involved in a positive-feedback loop that regulates $\mathrm{S} \mathrm{P}_{3}$ expression and signalling.

SK1 inhibitors--There are two major mechanisms of action of SK1 inhibitors. First, SK1 inhibitors (both catalytic and allosteric) can rapidly and reversibly inhibit catalytic activity 
(Baek et al., 2013). Second, SK1 inhibitors induce a longer term (e.g. 24 h) ubiquitinproteasomal degradation of SK1, thereby substantially reducing expression of the enzyme in cancer cells (Loveridge et al. 2010; Tonelli et al., 2010; Byun et al., 2013). Some SK1 inhibitors can be considered 'direct' inducers of the proteasomal degradation of SK1. An examples is PF-543, which binds to SK1 to induce a conformation that promotes its proteasomal degradation (Byun et al., 2013). Indeed, there is a strong correlation of concentration of inhibitor required to induce proteasomal degradation with the $K_{\mathrm{i}}$ for inhibition of catalysis. In contrast, a weak inhibitor of SK1/SK2, such as Ski (SKI-II, 4-[[4(4-chlorophenyl)-2-thiazolyl]amino]phenol), can induce activation of the proteasome to increase degradation of SK1 (Loveridge et al., 2010). This is an example of an 'indirect' inducer and occurs via an additional inhibition of dihydroceramide desaturase (Des1) (McNaughton et al., 2016), an enzyme within the de novo ceramide synthesis pathway, and which we propose restrains proteasomal activity.

Exosomal SIP receptor signalling in cancer-fibroblast microenvironment interactions--There is increasing evidence for a role for $\mathrm{S} 1 \mathrm{P}$ in regulation of the cancer-fibroblast microenvironment niche. In this regard, Albinet et al., (2014) reported that SK1 produces $\mathrm{S} 1 \mathrm{P}$, which is released from melanoma cells and which, in turn, promotes the differentiation of fibroblasts into myofibroblasts. SK1 in myofibroblasts then produces S1P, which promotes the $\mathrm{S} 1 \mathrm{P} / \mathrm{S}_{1} \mathrm{P}_{3}$-dependent metastatic spread of melanoma cells, thereby giving rise to bidirectional communication between tumour associated fibroblasts and melanoma cancer cells. In addition, Ponnusamy et al., (2012) have reported that host SK1 produces S1P, which promotes lung colonization and metastasis via an $\mathrm{S}_{1} \mathrm{P}_{2}$-dependent mechanism. Thus, reduced systemic S1P inhibits prostate cancer growth in transgenic adenocarcinoma of the mouse prostate $\left(\mathrm{TRAMP}^{+/+}\right) \mathrm{Sk}^{-/-}$mice or lung metastasis in $S p h k 1^{-/-}$mice. The loss of SK1 
promotes the expression of breast carcinoma metastasis suppressor 1 (Brms1) whereas S1P binding to $\mathrm{S}_{1} \mathrm{P}_{2}$ reduces Brms1 expression in these cancer cells (Ponnusamy et al., 2012). Taken together these studies indicate that host S1P (possibly involving stromal interaction) is capable of driving cancer metastasis. However, we describe here an additional, novel mechanism by which S1P receptors may regulate stromal-cancer interaction independently of $\mathrm{S} 1 \mathrm{P}$. In this regard, we have recently found that $\mathrm{S}_{1} \mathrm{P}_{2}(\mathrm{Mr}=42 \mathrm{kDa})$ is shed from MDA-MB231 breast cancer cells in exosomes present in conditioned medium (unpublished). We have

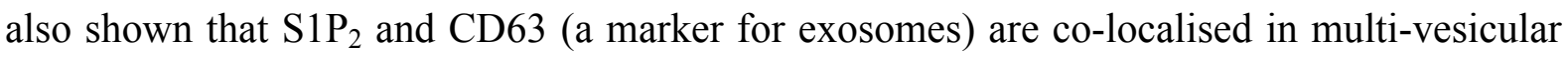
bodies (MVBs) in breast cancer cells (unpublished). Exosomal $\mathrm{S}_{1} \mathrm{P}_{2}$ is then proteolysed at the N-terminus when combined with fibroblasts to produce a smaller constitutively active form $(\mathrm{Mr}=38 \mathrm{kDa})$, which is taken up by the fibroblast. The short $\mathrm{S}_{1} \mathrm{P}_{2}$ form stimulates the ERK-1/2 pathway in fibroblasts and promotes proliferation of these cells. We have also shown that the activation of ERK-1/2 in fibroblasts in response to exosome or conditioned medium containing $\mathrm{S}_{1} \mathrm{P}_{2}$ is reduced by siRNA knockdown of $\mathrm{S}_{1} \mathrm{P}_{2}$ expression in breast cancer cells (unpublished). A schematic summarising this model for the role of $\mathrm{S}_{1} \mathrm{P}_{2}$ in cancer-fibroblast microenvironment is summarised in Figure 4.

Topographical arrangement of a GPCR in exosomes and uptake into the plasma-membrane of recipient cells would place the C-terminal tail of the GPCR on the cytoplasmic side of the recipient cell. This might enable the short form of $\mathrm{S}_{1} \mathrm{P}_{2}$ to initiate signalling into the intracellular compartment of the recipient cell. Others have shown that extracellular vesicles derived from immortalized human mammary luminal epithelial HB4a and C5.2 cells contain $\mathrm{S}_{1} \mathrm{P}_{3}$ and $\mathrm{S}_{\mathrm{P}}$, although the functional significance was not evaluated (Amorim et al., 2014). Constitutive activation of $\mathrm{S}_{2} \mathrm{P}_{2}$ could occur as a consequence of proteolysis at the Nterminus, which might normally exert an inhibitory constraint on $\mathrm{S}_{1} \mathrm{P}_{2}$ signalling. Indeed, $\mathrm{N}$ - 
terminal proteolysis of GPCRs has been shown to modulate signalling. For instance, the thyroid-stimulating hormone (TSH) receptor is cleaved by a metalloproteinase at the $\mathrm{N}$ terminus to produce a form of the receptor that is constitutively active by removal of an inhibitory N-terminal domain (Couet et al., 1996; Zhang et al., 2000).

There are several examples of communication between cancer cells and recipient cells involving exosomes. For example, exosomes derived from nasopharyngeal carcinoma contain latent membrane protein 1 (LMP1), which can induce ERK and AKT activation in recipient cells (Meckes et al., 210). In addition, exosomes derived from mesenchymal stem cells (MSC) promote MCF-7 migration via a Wnt-dependent mechanism of breast cancer cells (Lin et al., 2013). Lin et al., (2016) have also reported that bone marrow-derived mesenchymal stromal cells release exosomes to promote tumour growth.

The functional consequence of the transfer of $\mathrm{S} \mathrm{P}_{2}$ from breast cancer cells to fibroblasts is to induce expansion (e.g. increased DNA synthesis and proliferation) of the fibroblast population, which is required to initiate and sustain breast cancer metastatic spread. Future studies will require characterisation of the signalling properties of the short $\mathrm{S}_{1} \mathrm{P}_{2}$ receptor in order to determine its role (if any) in metastatic spread. This can be explored by overexpression of the short $\mathrm{S}_{1} \mathrm{P}_{2}$ in fibroblasts in vivo in order to establish the effect on metastatic spread in breast cancer xenograft mouse models.

Conclusion--Our studies have provided a mechanistic model for how SK1 can translocate and localise with the plasma-membrane to access substrate for production of S1P. This process is critical for cancer progression, dependent on S1P, which acts intracellularly and/or by binding to $\mathrm{S} 1 \mathrm{P}$ receptors. In addition, we have provided novel evidence for a role of 
constitutively active $\mathrm{S}_{1} \mathrm{P}_{2}$ in cancer-fibroblast interactions that might reveal S1P-independent actions of this receptor. Therefore, targeted therapeutics aimed at inhibiting SK1 activity and/or promoting its proteasomal degradation and/or inhibiting its translocation to the plasma-membrane may offer novel approaches to preventing cancer cell survival. Alternatively, inverse agonists of $\mathrm{S} 1 \mathrm{P}_{2}$ might exhibit additional therapeutic utility in terms of blocking cancer-fibroblast interactions to prevent metastatic spread.

\section{Acknowledgements}

Ashref El Buri received funding for a PhD studentship at SU from the Libyan Government.

\section{References}

Adams, D.R., Pyne, S., Pyne, N.J., 2016. Sphingosine Kinases: Emerging Structure-Function Insights. Trends Biochem. Sci. 41, 395-409.

Albinet, V., Bats, M.L., Huwiler, A., Rochaix, P., Chevreau, C., Ségui, B., Levade, T., Andrieu-Abadie, N., 2014. Dual role of sphingosine kinase-1 in promoting the differentiation of dermal fibroblasts and the dissemination of melanoma cells. Oncogene. 33, 3364-3373.

Amorim, M., Fernandes, G., Oliveira, P., Martins-de-Souza, D., Dias-Neto, E., Nunes, D., 2014. The overexpression of a single oncogene (ERBB2/HER2) alters the proteomic landscape of extracellular vesicles. Proteomics 14, 1472-1479.

Baek, D.J., MacRitchie, N., Anthony, N.G., Mackay, S.P., Pyne, S., Pyne, N.J., 2013. Bittman R. Structure-activity relationships and molecular modeling of sphingosine kinase inhibitors. J. Med. Chem. 56, 9310-9327. 
Barr, R.K., Lynn, H.E., Moretti, P.A., Khew-Goodall, Y., Pitson, S.M. 2008. Deactivation of sphingosine kinase 1 by protein phosphatase 2A. J. Biol. Chem. 283, 34994-35002.

Byun, H.S., Pyne, S., Macritchie, N., Pyne, N.J., Bittman, R., 2013. Novel sphingosinecontaining analogues selectively inhibit sphingosine kinase (SK) isozymes, induce SK1 proteasomal degradation and reduce DNA synthesis in human pulmonary arterial smooth muscle cells. Medchemcomm. 4(10).

Chan, J.P., Hu, Z., Sieburth, D., 2012. Recruitment of sphingosine kinase to presynaptic terminals by a conserved muscarinic signalling pathway promotes neurotransmitter release. Genes Dev. 26, 1070-1085.

Couet, J., Sar, S., Jolivet, A., Hai, M.T., Milgrom, E., Misrahi, M., 1996. Shedding of human thyrotropin receptor ectodomain. Involvement of a matrix metalloprotease. J. Biol. Chem. $271,4545-4552$.

Galvani, S., Sanson, M., Blaho, V.A., Swendeman, S.L., Obinata, H., Conger, H., Dahlbäck, B., Kono, M., Proia, R.L., Smith, J.D., Hla T., 2015. HDL-bound sphingosine 1-phosphate acts as a biased agonist for the endothelial cell receptor $\mathrm{S}_{1} \mathrm{P}_{1}$ to limit vascular inflammation. Sci. Signal. 8(389):ra79.

Gustin, D.J., Li, Y., Brown, M.L., Min, X., Schmitt, M.J., Wanska, M., Wang, X., Connors, R,, Johnstone, S., Cardozo, M., Cheng, A.C., Jeffries, S., Franks, B., Li, S., Shen, S., Wong, M., Wesche, H., Xu, G., Carlson, T.J., Plant, M., Morgenstern, K., Rex, K., Schmitt, J., 
Coxon, A,, Walker, N., Kayser, F., Wang, Z., 2013. Structure guided design of a series of sphingosine kinase (SphK) inhibitors. Bioorg. Med. Chem. Lett. 23, 4608-4016.

Hengst, J.A., Guilford, J.M., Fox, T.E., Wang, X., Conroy, E.J., Yun, J.K., 2009. Sphingosine kinase 1 localized to the plasma membrane lipid raft microdomain overcomes serum deprivation induced growth inhibition. Arch. Biochem. Biophys. 492, 62-73.

Hengst, J.A., Guilford, J.M., Conroy, E.J., Wang, X., Yun, J.K., 2010. Enhancement of sphingosine kinase 1 catalytic activity by deletion of 21 amino acids from the $\mathrm{COOH}$ terminus. Arch. Biochem. Biophys. 494, 23-31.

Jarman, K.E., Moretti, P.A., Zebol, J.R., Pitson, S.M., 2010. Translocation of sphingosine kinase 1 to the plasma membrane is mediated by calcium- and integrin-binding protein 1 . J. Biol. Chem. 285, 483-492.

Lim, K.G., Tonelli, F., Li, Z., Lu, X., Bittman, R., Pyne, S., Pyne, N.J., 2011. FTY720 analogues as sphingosine kinase 1 inhibitors: enzyme inhibition kinetics, allosterism, proteasomal degradation, and actin rearrangement in MCF-7 breast cancer cells. J. Biol. Chem. 286, 18633-18640.

Lin, R., Wang, S., Zhao, R.C., 2013. Exosomes from human adipose-derived mesenchymal stem cells promote migration through Wnt signalling pathway in a breast cancer cell model. Mol. Cell Biochem. 383, 13-20. 
Lin, L.Y., Du, L.M., Cao, K., Huang, Y., Yu, P.F., Zhang, L.Y., Li, F.Y., Wang, Y., Shi, Y.F., 2016. Tumour cell-derived exosomes endow mesenchymal stromal cells with tumourpromotion capabilities. Oncogene 35, 6038-6042.

Long, J.S., Edwards, J., Watson, C., Tovey, S., Mair, K.M., Schiff, R., Natarajan, V., Pyne, N.J., Pyne, S., 2010. Sphingosine kinase 1 induces tolerance to human epidermal growth factor receptor 2 and prevents formation of a migratory phenotype in response to sphingosine 1-phosphate in estrogen receptor-positive breast cancer cells. Mol. Cell Biol. 30, 3827-3841.

Loveridge, C., Tonelli, F., Leclercq, T., Lim, K.G., Long, J.S., Berdyshev, E., Tate, R.J., Natarajan, V., Pitson, S.M., Pyne, N.J., Pyne, S., 2010. The sphingosine kinase 1 inhibitor 2(p-hydroxyanilino)-4-(p-chlorophenyl)thiazole induces proteasomal degradation of sphingosine kinase 1 in mammalian cells. J. Biol. Chem. 285, 38841-38852.

Maczis, M., Milstien, S., Spiegel, S., 2016. Sphingosine-1-phosphate and estrogen signaling in breast cancer. Adv. Biol. Regul. 60, 160-165.

McNaughton, M., Pitman, M., Pitson, S.M., Pyne, N.J., Pyne, S., 2016, Proteasomal degradation of sphingosine kinase 1 and inhibition of dihydroceramide desaturase by the sphingosine kinase inhibitors, SKi or ABC294640, induces growth arrest in androgenindependent LNCaP-AI prostate cancer cells. Oncotarget 7, 16663-16675.

Meckes, D.G. Jr, Shair, K.H., Marquitz, A.R., Kung, C.P., Edwards, R.H., Raab-Traub, N., 2010. Human tumor virus utilizes exosomes for intercellular communication. Proc Natl Acad Sci (U S A.) 107, 20370-20375. 
Ohotski, J., Edwards, J., Elsberger, B., Watson, C., Orange, C., Mallon, E., Pyne, S., Pyne, N.J., 2013. Identification of novel functional and spatial associations between sphingosine kinase 1, sphingosine 1-phosphate receptors and other signalling proteins that affect prognostic outcome in estrogen receptor-positive breast cancer. Int. J. Cancer. 132, 605-616.

Olivera, A., Rosenthal, J., Spiegel, S., 1996. Effect of acidic phospholipids on sphingosine kinase. J. Cell Biochem. 60, 529-537.

Pham, D.H., Powell, J.A., Gliddon, B.L., Moretti, P.A., Tsykin, A., Van der Hoek, M., Kenyon, R., Goodall, G.J., Pitson, S.M., 2014. Enhanced expression of transferrin receptor 1 contributes to oncogenic signalling by sphingosine kinase 1 . Oncogene $33,5559-5568$.

Pitson, S.M., Moretti, P.A., Zebol, J.R., Lynn, H.E., Xia, P., Vadas, M.A., Wattenberg. B.W., 2003. Activation of sphingosine kinase 1 by ERK1/2-mediated phosphorylation. EMBO J. $22,5491-5500$.

Pitson, S.M., Xia, P., Leclercq, T.M., Moretti, P.A., Zebol, J.R., Lynn, H.E., Wattenberg, B.W., Vadas, M.A., 2005. Phosphorylation-dependent translocation of sphingosine kinase to the plasma membrane drives its oncogenic signalling. J. Exp. Med. 201, 49-54.

Ponnusamy, S., Selvam, S.P., Mehrotra, S., Kawamori, T., Snider, A.J., Obeid, L.M., Shao, Y., Sabbadini, R., Ogretmen, B., 2012. Communication between host organism and cancer cells is transduced by systemic sphingosine kinase 1/sphingosine 1-phosphate signalling to regulate tumour metastasis. EMBO Mol. Med. 4, 761-775. 
Pyne, S., Adams, D.R., Pyne, N.J., 2016. Sphingosine 1-phosphate and sphingosine kinases in health and disease: Recent advances. Prog Lipid Res. 62, 93-106.

Pyne, N.J., McNaughton, M., Boomkamp, S., MacRitchie, N., Evangelisti, C., Martelli, A.M., Jiang, H.R., Ubhi, S., Pyne, S., 2016/ Role of sphingosine 1-phosphate receptors, sphingosine kinases and sphingosine in cancer and inflammation. Adv Biol. Regul. 60, 151159.

Pyne N.J., Pyne S., 2010. Sphingosine 1-phosphate and cancer. Nat Rev Cancer. 10, 489-503.

Shen, H., Giordano, F., Wu, Y., Chan, J., Zhu, C., Milosevic, I., Wu, X., Yao, K., Chen, B., Baumgart, T., Sieburth, D., De Camilli, P., 2014. Coupling between endocytosis and sphingosine kinase 1 recruitment. Nat. Cell Biol. 16, 652-662.

Stahelin, R.V., Hwang, J.H., Kim, J.H., Park, Z.Y., Johnson, K.R., Obeid, L.M., Cho, W., 2005. The mechanism of membrane targeting of human sphingosine kinase 1. J. Biol. Chem. $280,43030-43038$.

Sutherland, C.M., Moretti, P.A., Hewitt, N.M., Bagley, C.J., Vadas, M.A., Pitson, S.M., 2006. The calmodulin-binding site of sphingosine kinase and its role in agonist-dependent translocation of sphingosine kinase 1 to the plasma membrane. J. Biol. Chem. 281, 1169311701. 
Takabe, K., Spiegel, S., 2014. Export of sphingosine-1-phosphate and cancer progression. J. Lipid Res. 55, 1839-1846.

ter Braak, M., Danneberg, K., Lichte, K., Liphardt, K., Ktistakis, N.T., Pitson, S.M., Hla, T., Jakobs, K.H., Meyer zu Heringdorf, D., 2009. Galpha(q)-mediated plasma membrane translocation of sphingosine kinase-1 and cross-activation of S1P receptors. Biochim. Biophys. Acta. 1791, 357-370.

Tonelli, F., Lim, K.G., Loveridge, C., Long, J., Pitson, S.M., Tigyi, G., Bittman, R., Pyne, S., Pyne, N.J., 2010. FTY720 and (S)-FTY720 vinylphosphonate inhibit sphingosine kinase 1 and promote its proteasomal degradation in human pulmonary artery smooth muscle, breast cancer and androgen-independent prostate cancer cells. Cell Signal. 22, 1536-1542.

Wang, J., Knapp, S., Pyne, N.J., Pyne, S., Elkins, J.M., 2014b. Crystal Structure of Sphingosine Kinase 1 with PF-543. ACS Med. Chem. Lett. 5, 1329-1333.

Wang, Q., Li, J., Li, G., Li, Y., Xu, C., Li, M., Xu, G., Fu, S., 2014a. Prognostic significance of sphingosine kinase 2 expression in non-small cell lung cancer. Tumour Biol. 2014. 35, $363-368$

Wang, Z., Min, X., Xiao, S.H., Johnstone, S., Romanow, W., Meininger, D., Xu, H., Liu, J., Dai, J., An, S., Thibault, S., Walker, N., 2013. Molecular basis of sphingosine kinase 1 substrate recognition and catalysis. Structure 21, 798-809. 
Watson, C., Long, J.S., Orange, C., Tannahill, C.L., Mallon, E., McGlynn, L.M., Pyne, S., Pyne, N.J., Edwards, J., 2010. High expression of sphingosine 1-phosphate receptors, S1P 1 and $\mathrm{S}_{1} \mathrm{P}_{3}$, sphingosine kinase 1 , and extracellular signal-regulated kinase- $1 / 2$ is associated with development of tamoxifen resistance in estrogen receptor-positive breast cancer patients. Am. J. Pathol. 177, 2205-2215.

Vadas, M., Xia, P., McCaughan, G., Gamble, J., 2008. The role of sphingosine kinase 1 in cancer: oncogene or non-oncogene addiction? Biochim. Biophys. Acta. 1781, 442-447.

Xia, P., Gamble, J.R., Wang, L., Pitson, S.M., Moretti, P.A., Wattenberg, B.W., D'Andrea, R.J., Vadas, M.A., 2000. An oncogenic role of sphingosine kinase. Curr. Biol. 10, 1527-1530.

Xia, P,, Wang, L., Moretti, P.A., Albanese, N., Chai, F., Pitson, S.M., D'Andrea, R.J., Gamble, J.R., Vadas, M.A. 2002. Sphingosine kinase interacts with TRAF2 and dissects tumor necrosis factor-alpha signalling. J. Biol. Chem. 277, 7996-8003.

Zhang, M., Tong, K.P., Fremont, V., Chen, J., Narayan, P., Puett, D., Weintraub, B.D., Szkudlinski, M.W., 2000. The extracellular domain suppresses constitutive activity of the transmembrane domain of the human TSH receptor: implications for hormone-receptor interaction and antagonist design. Endocrinology 141, 3514-3517.

\section{Figure Legends}

Figure 1. (Left) SK1 tertiary structure illustrated with co-crystal structure 3VZB (chain A) with sphingosine (Sph; blue surface) occupying the lipid-binding J-channel of the C-terminal 
domain (CTD); Mg-ADP (green sphere/pink surface) is superimposed from a separate SK1 co-crystal structure (3VZD chain C) (Wang et al., 2013). The regulatory R-loop encompassing the ERK1/2 phosphorylation site (Ser225, circled) is fully ordered here, but exhibits partial disorder in other chains and crystal structures (especially in the N-terminal half including the phosphoacceptor site). None of the constructs from which the currently available SK1 crystal structures have been obtained encompass the C-terminal 20-residue sequence ( $c f$. Figure 3), a sequence that facilitates protein binding and that also mediates additional regulatory control (Xia et al., 2002; Barr et al., 2008) (Inset, right). Viewed as left, but highlighting detail of the catalytic centre of SK1. Five motifs (motif-1 to motif-5) contribute to binding of the nucleotide. The 'P-loop' (motif-3, blue dotted ribbon) and 'Tloop' (motif-4, red dotted ribbon) engage the $\alpha$ - and $\beta$-phosphates of the nucleotide. P-loop residue Asp81 caps the T-loop and also serves as the base for deprotonation of the Sph-1-OH to facilitate transfer of the $\gamma$-phosphate from ATP during catalytic turnover. Comparison of SK1 crystal structures with and without bound nucleotide show significant changes in the conformation of T-loop residues and suggests that plasticity may be important for catalytic function, in particular to allow backbone hydrogen donor interactions to track the nucleotide $\beta$-phosphate on separation from the $\gamma$-phosphate of ATP. In the ADP bound structure, the $\beta$ phosphate is engaged by two arginines, R185 and R191 (marked), from lipid binding loop-1 (LBL-1). However, Arg191 is also suitably positioned to interact with the $\gamma$-phosphate of ATP and to stabilise the S1P product resulting from catalytic turnover. Much of the binding affinity for Sph derives from hydrophobic surface and van der Waals contact in the J-channel, but the lipid is also held by a highly organised network of polar interactions engaging the head unit, prominent among which is the hydrogen bonded interaction of the Sph-3-OH with the LBL-1 residue, Asp178 (marked). 
Figure 2. Hypothetical relay mechanism for regulatory control mediated by phosphorylation of Ser225 (Adams et al., 2016). SK1 crystal structures suggest that in the enzyme basal state the tip of the R-loop (green ribbon) interfaces with residues (93-ERPDW-97) located at the $\alpha$ $3 / 4$ junction (orange ribbon) in the N-terminal domain (NTD). Maintenance of this interface is likely dependent on insertion of R-loop aspartate, D235 (marked), into a basic pocket lined by His156/Arg162/His355 (not marked). Displacement of D235 from this site by phosphoSer225 (solid black arrow) would disengage the R-loop tip from the 93-ERPDW-97 sequence (blue arrows). This may perturb NTD residues such as glutamate E93 so as to modulate an adjacent region of positive electrostatic surface potential (blue ellipse) that might be involved in targeting of SK1 to phosphatidylserine (PS)-enriched membrane environments (cf. Figure 3). Two residues for which mutation disrupts PS engagement are Thr54 and Asn89 (marked).

Figure 3. Membrane engagement hypothesis for dimeric SK1 (Adams et al., 2016). (A) Dimeric assembly of SK1 according to the crystallographically observed mode places the two LBL-1 loops (cyan ribbon) on the same face of the rod-like dimer structure and on the opposite side of the protein to the regulatory R-loop (green ribbon). The dimeric SK1 structure, viewed orthogonally in panel A(ii), may be topologically suited for binding to membranes that are enriched in acidic phospholipids (phosphatidylserine, PS) and that exhibit strong positive curvature. Interaction of membrane PS with a positively charged dimer groove would require insertion of the hydrophobic LBL-1 islands into the membrane, consistent with the known requirement of F197 and L198 (marked) for membrane binding (Shen et al., 2014). Opening and closure of the flap-like LBL-1 structures (black arrows) may allow extraction of sphingosine (Sph) substrate from the membrane for gated entry into the enzyme's J-channel. The C-terminal tail sequence (dotted black line), which facilitates protein binding and plays a regulatory role (Xia et al., 2002; Barr et al., 2008; Hengst et al. 
2010), is missing from available SK1 crystal structures but would be connected at Gly364 (black spheres) on either side of the dimer structure. The connection site is proximal to the C-terminal end of helix- $\alpha 5$ (orange ribbon, marked in panel A) and may allow for cappingmodulated regulatory control of catalytic turnover by the tail sequence due to leverage on the T-loop (also marked in panel A). In the absence of bound protein or phosphorylation on Rloop Ser225, the tail sequence may potentially fold to obstruct dimerization and/or membrane engagement. (B) Qualitative electrostatic potential surface mapping reveals a groove with significant net positive charge (dashed box) that aligns parallel with the axes of two $\alpha 8$ helices from the dimer LBL-1 loops. Prominent hydrophobic islands (cyan circles) are generated by exposed hydrophobic residues from helices $\alpha 8$ (including F197 and L198). We postulate that it is the combination of these features with their relative topology in the dimer that may confer the enzyme's particular membrane localisation determinants.

Figure 4 Schematic model for the release of $\mathrm{S} \mathrm{P}_{2}$ in exosomes from cancer cells and possible interaction with fibroblasts. $\mathrm{S}_{1} \mathrm{P}_{2}$ is endocytosed and processed into MVBs in MDA-MB-231 cells whereupon it is released in exosomes. We propose that exosomal $\mathrm{S}_{1} \mathrm{P}_{2}$ is taken up and processed by fibroblasts to a shorter constitutively active form, lacking the $\mathrm{N}$ terminus (red), where it can activate the ERK pathway to promote proliferation and, in turn, release factors that promote metastatic spread. 

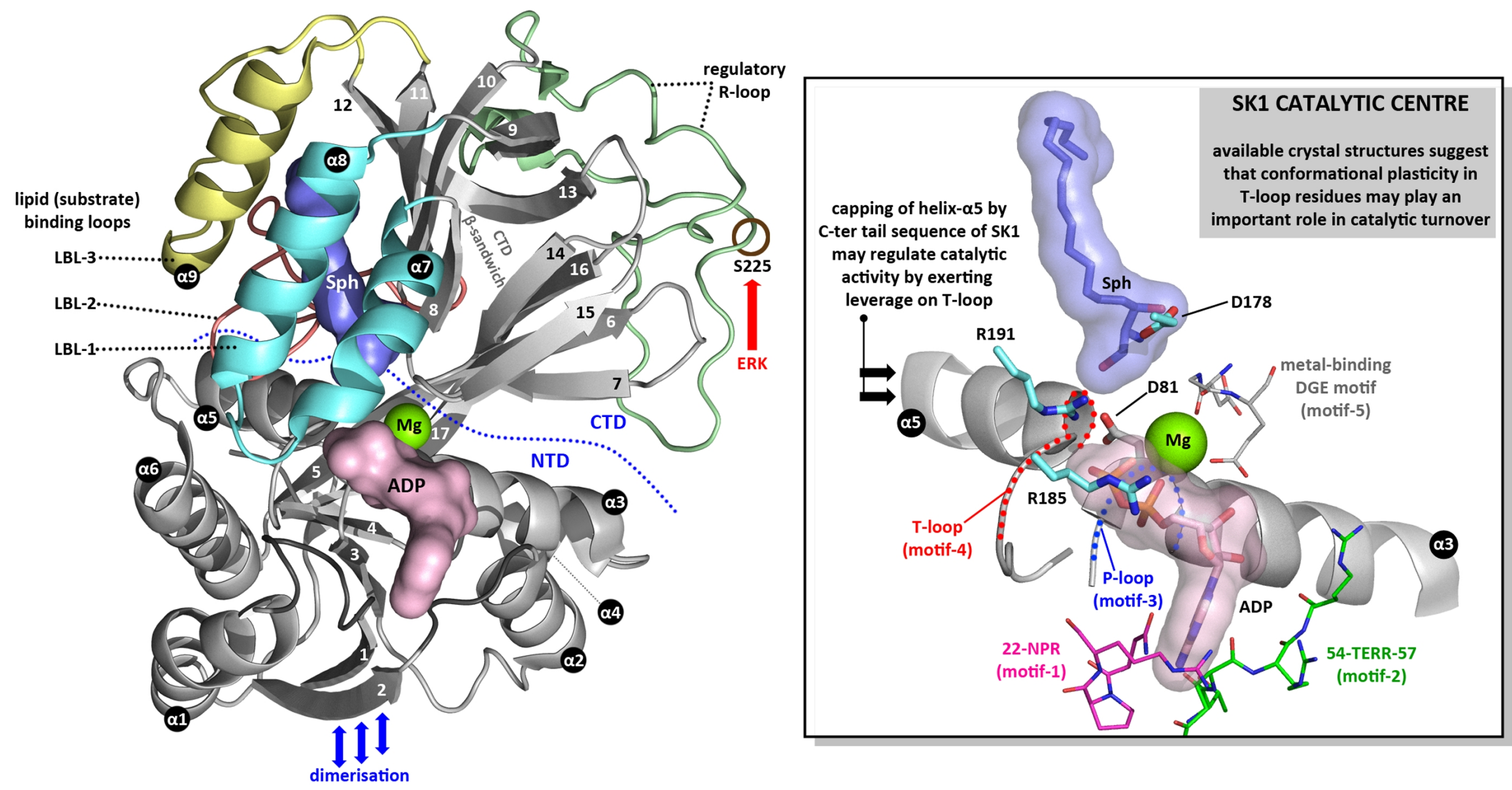


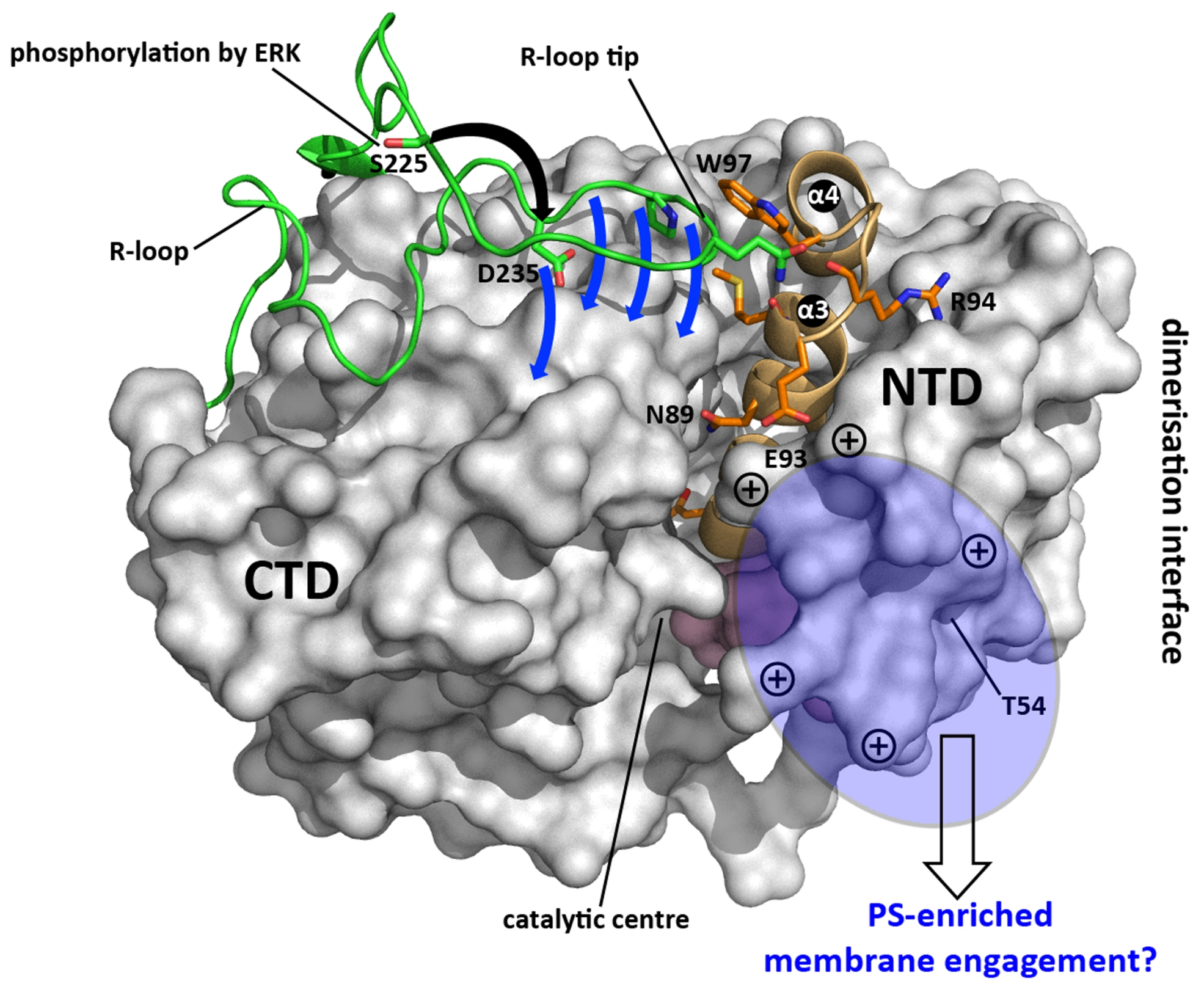


A (i)

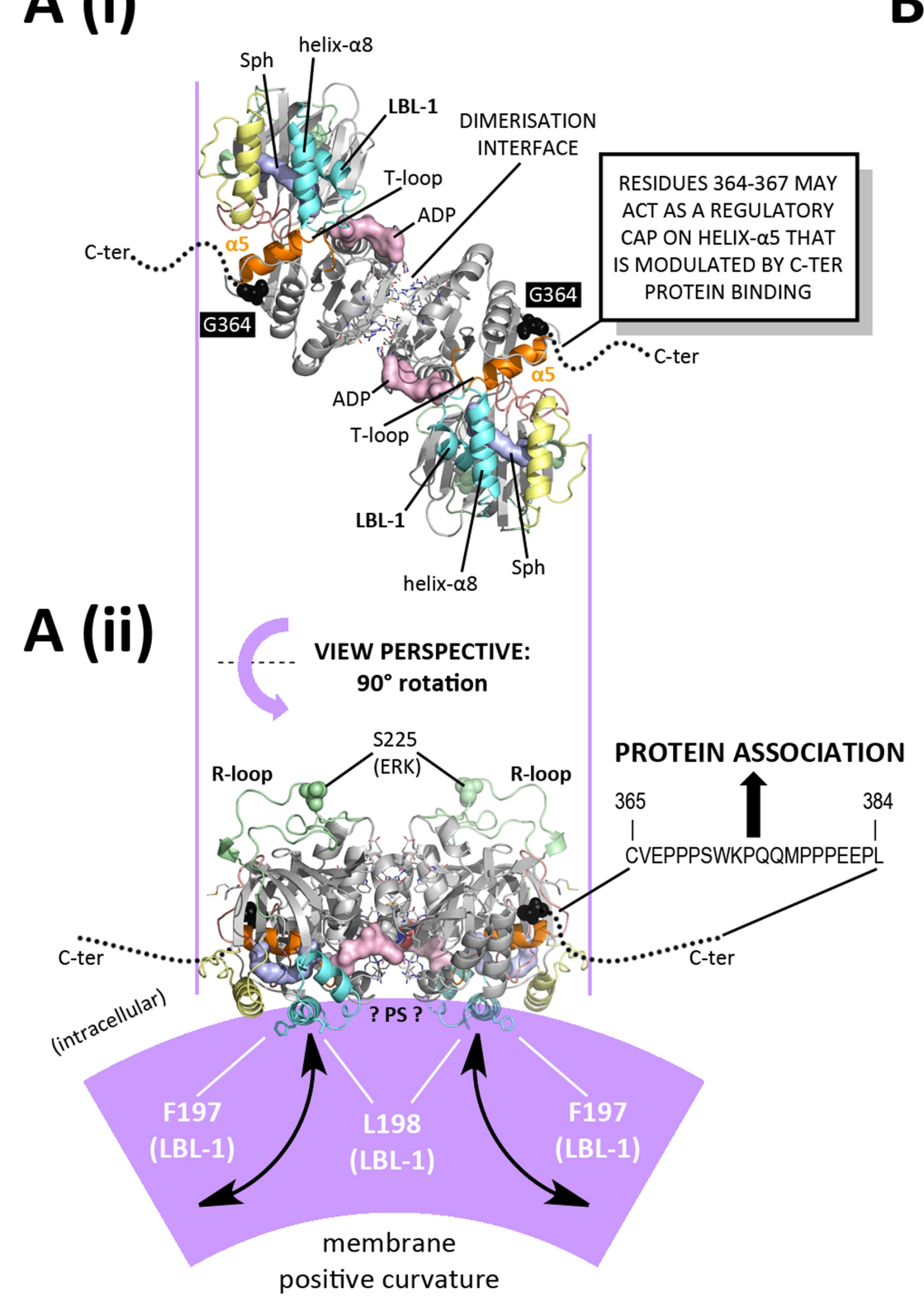

B VIEW PERSPECTIVE: as left

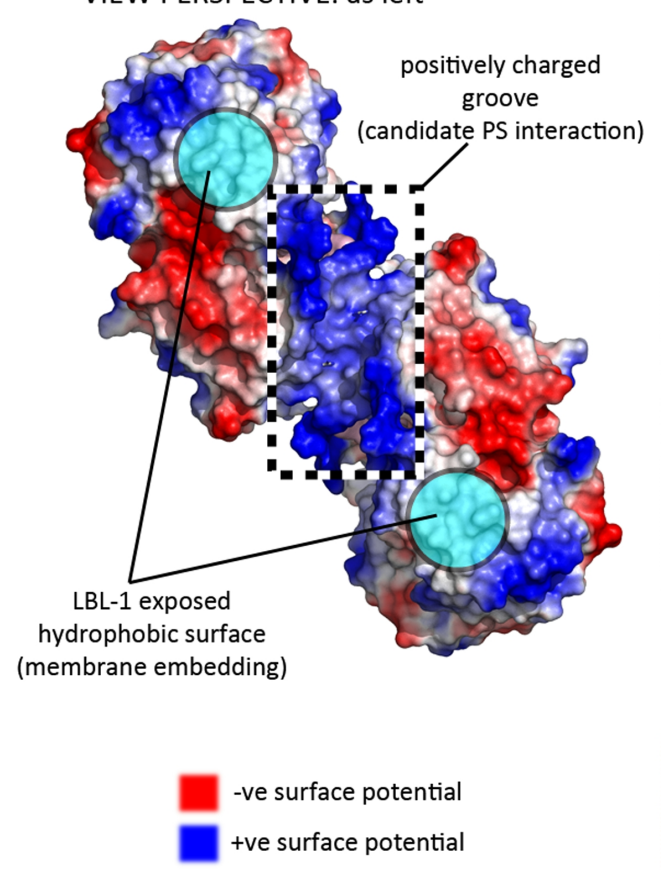




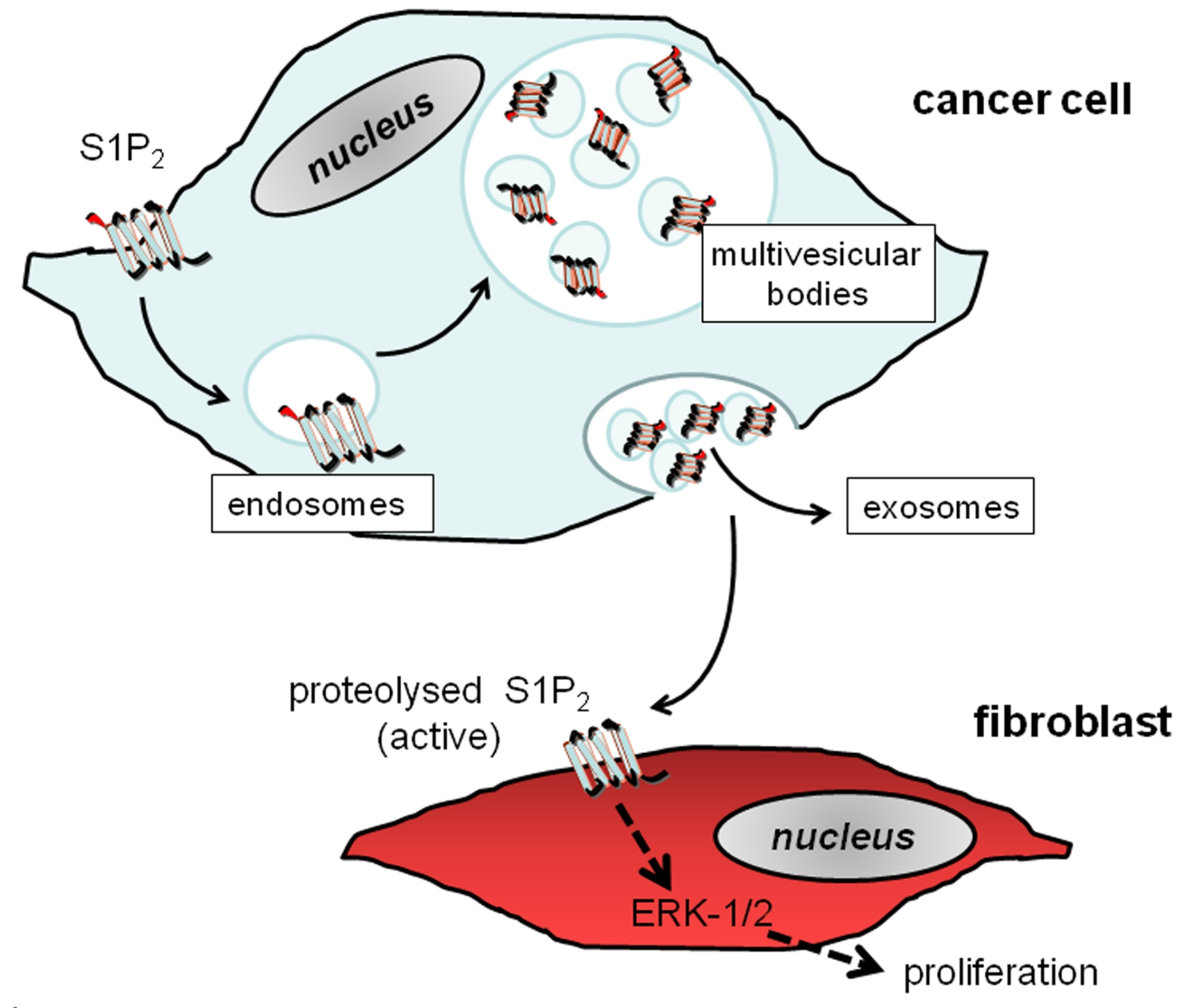

Figure 4 